\title{
Effects of Xiaochaihu decoction on the expression of cytochrome P450s in rats
}

\author{
HONGFANG LI $^{1-3 *}$, YUNYAN TANG ${ }^{1,4^{*}}$, WEIPENG WEI ${ }^{1-3}$, CHENGCHEN YIN $^{1-3}$ and FUSHAN TANG ${ }^{1-3}$ \\ ${ }^{1}$ Department of Clinical Pharmacy, Key Laboratory of Basic Pharmacology of Guizhou Province and School of Pharmacy; \\ ${ }^{2}$ Key Laboratory of Basic Pharmacology of Ministry of Education and Joint International Research Laboratory of \\ Ethnomedicine of Ministry of Education; ${ }^{3}$ Key Laboratory of Clinical Pharmacy of Zunyi City, Zunyi Medical University, \\ Zunyi, Guizhou 563000; ${ }^{4}$ Department of Pharmacy, Meitan People's Hospital, Zunyi, Guizhou 564100, P.R. China
}

Received July 16, 2020; Accepted December 15, 2020

DOI: $10.3892 /$ etm. 2021.10020

\begin{abstract}
Xiaochaihu decoction is one of the most important traditional Chinese medicines that is widely used with other drugs in clinical practice, and may cause drug-drug interactions. However, there is not sufficient experimental evidence for the effects of Xiaochaihu decoction on cytochrome P450s (CYPs). The aim of the present study was to investigate the effects of Xiaochaihu decoction on the mRNA and protein levels of hepatic CYPs. Eighty normal male Sprague-Dawley (SD) rats were randomly divided into two groups based on body weight and duration of drug administration (3 and 6 days). Each group was further divided into subgroups: Control group $(2 \mathrm{ml}$ $5 \%$ CMC-Na); hepatic enzyme inducer group $(50 \mathrm{mg} / \mathrm{kg} /$ day rifampicin); and experimental groups (Xiaochaihu decoction: Low dose, $1.7 \mathrm{~g} / \mathrm{kg} /$ day; medium dose, $3.4 \mathrm{~g} / \mathrm{kg} /$ day; high dose, $6.8 \mathrm{~g} / \mathrm{kg} /$ day). The effects of Xiaochaihu decoction on Cypla2, Cyp3al, Cyp2d6, and Cyplbl mRNA and protein expression in rats were evaluated using reverse transcription quantitative reverse transcription polymerase chain reaction and western blot analysis. After 3 days, medium dose of Xiaochaihu decoction inhibited the mRNA and protein expression of Cypla2, Cyp3al and Cyplbl. In addition, after 6 days, Xiaochaihu decoction induced Cyp3al mRNA expression at low and medium doses; Cyp2d6 mRNA expression at low and high doses; and Cyp $2 d 6$ protein expression at high doses. Nonetheless, the gene and protein expression of Cyplbl was not affected at any dose. The findings of the present study may provide insights into potential drug-drug interactions associated with Xiaochaihu decoction.
\end{abstract}

Correspondence to: Professor Fushan Tang, Department of Clinical Pharmacy, Key Laboratory of Basic Pharmacology of Guizhou Province and School of Pharmacy, Zunyi Medical University, 6 Xuefu West Road, Zunyi, Guizhou 563000, P.R. China E-mail: fstang@vip.163.com

${ }^{*}$ Contributed equally

Key words: Xiaochaihu decoction, cytochrome P450, drug-drug interaction, drug metabolism, traditional Chinese medicines

\section{Introduction}

Xiaochaihu decoction is a traditional Chinese medicine that is prepared from seven herbs (Bupleuri Radix, Pinelliae Tuber, Scutellariae Radix, Zizyphi Fructus, Ginseng Radix, Glycyrrhizae Radix, Zingiberis Rhizoma) (1), which has attracted increasing attention as an alternative treatment and supplement. Xiaochaihu decoction has been widely applied for the treatment of 15 types of 262 diseases, including diseases of the digestive, respiratory and nervous system (2). To facilitate the use of Xiaochaihu decoction in the clinical setting, researchers have developed several dosage forms of Xiaochaihu decoction, including granules, capsules, tablets and effervescent tablets, which have been recorded in the 2015 edition of Chinese Pharmacopoeia (Part I) (3). With continuous experimental and clinical exploration, Xiaochaihu decoction has been co-administered with various synthesised drugs such as rabeprazole, isosorbide mononitrate, and entecavir for digestive, cardiovascular, cerebrovascular and immune diseases, respectively (4-6).

The concomitant administration of two or more drugs may lead to drug-drug interactions (DDIs), which are considered a common cause of adverse drug reactions (ADRs) (7). Previous studies have reported that co-administration of Xiaochaihu decoction with tolbutamide (8), carbamazepine (9) and cyclosporine A (CsA) (10) affects the in vivo efficacy of the latter drugs. The interaction between Xiaochaihu decoction and other concomitant drugs is a problem worthy of attention for basic and clinical researchers.

Despite many reasons for DDIs, metabolic interactions account for $\sim 40 \%$ of them. Metabolic interactions are often caused by enzymes involved in drug metabolism, mainly hepatic enzymes in the cytochrome P450 (CYP) family. CYPs are mixed functional oxidases involved in the metabolism of endogenous and exogenous substances $(11,12)$, and they can significantly affect drug metabolism (13). Many drugs act as substrates, inducers or inhibitors of CYPs. When a drug induces or inhibits CYPs, it causes changes in the physiological environment of another drug, consequently altering their efficacy and even leading to toxicity (14). Therefore, changes in the content or activity of CYPs by one or more concomitant drugs are one of the main causes of DDIs in the 
clinical setting. It is important to study the effects of drugs on CYPs for understanding the interactions between drugs used in combination.

With the continuous development as well as improvements in the knowledge and understanding of traditional Chinese medicine, Xiaochaihu decoction and other formulations would be increasingly used in combination with synthesised drugs. Xiaochaihu decoction in combination with other drugs may alter the metabolism of the concomitant drug and lead to changes in the blood levels of the drugs. It may further decrease the efficacy of drugs or increase the likelihood of ADRs. To effectively obtain the benefits and avoid the disadvantages of combination therapy to the utmost, it is necessary to identify and confirm the mechanisms underlying these interactions at the earliest. At present, there is not sufficient experimental information on the effects of Xiaochaihu decoction on CYPs. Therefore, further research is needed to confirm the existing conclusions. Modern pharmacological toxicology studies have shown that many drugs affect the gene transcription and protein expression of CYPs $(14,15)$. Therefore, rats were selected to investigate the effects of Xiaochaihu decoction on gene transcription and protein expression of different subtypes of CYPs. The findings will help better elucidate the effects of combining Xiaochaihu decoction with other drugs that are metabolised by CYPs.

\section{Materials and methods}

Medicinal materials and reagents. The crude components of Xiaochaihu decoction, including Bupleuri Radix, Pinelliae Tuber, Scutellariae Radix, Zizyphi Fructus, Ginseng Radix, Glycyrrhizae Radix, and Zingiberis Rhizoma, were all purchased from the Department of Pharmacy in the Affiliated Hospital of Zunyi Medical University (Guizhou, China) and identified as quality Chinese herb medicines by Professor Jianwen Yang in Department of Pharmacognosy, Zunyi Medical University. Rifampicin was purchased from Chengdu ALFA Biotechnology Co., Ltd. Baicalin was purchased from Guizhou Dida Technology Co., Ltd. All other reagents were obtained from local reagent companies.

Preparation of Xiaochaihu decoction. Xiaochaihu decoction was prepared according to the proportion of Xiaochaihu granules in the Pharmacopoeia of the People's Republic of China 2015 edition (3) and the optimal extraction scheme of Xiaochaihu decoction reported by Cai et al (16). Briefly, $125 \mathrm{~g}$ Bupleuri Radix, 45 g Pinelliae Tuber, 45 g Scutellariae Radix, 45 g Zizyphi Fructus, 45 g ginger, 45 g Codonopsis Pilosula and $45 \mathrm{~g}$ Glycyrrhizae Radix were mixed with an 8-fold volume of distilled water evenly. After the herbs were moistened thoroughly, the mixture was boiled at $100^{\circ} \mathrm{C}$ for $40 \mathrm{~min}$ and filtered through a gauze to obtain the filtrate. Subsequently, the dregs were boiled with an 8 -fold volume of distilled water at $100^{\circ} \mathrm{C}$ for $40 \mathrm{~min}$ and filtered again. Subsequently, the filtrates were mixed together and concentrated to a brown sticky extract $(1 \mathrm{~g} / \mathrm{ml})$ in a rotary evaporator to obtain the decoction for experiments. The decoctions were stored at $4^{\circ} \mathrm{C}$.

Rifampicin solution. Rifampicin powder was suspended in distilled water and mixed in a vortex mixer to obtain rifampicin at a concentration of $5 \mathrm{mg} / \mathrm{ml}$ prior to each administration.
Animals. Eighty specific pathogen-free (SPF) male SD rats $(220 \pm 20 \mathrm{~g})$ for the experiments were provided by Liaoning Changsheng Biotechnology Co., Ltd. [Animal license number of the rats was SCXK: (Liaoning) 2015-0001]. All the rats were housed in environmentally controlled conditions (temperature, $20-24^{\circ} \mathrm{C}$; and relative humidity, $40-60 \%$ ) with a $12-\mathrm{h}$ light/dark cycle. Rats were acclimated to the environment for 7 days before the experiments. All animal experiments were strictly carried out in accordance with the NIH guidelines for the Care and Use of Laboratory Animals (NIH publications no. 80-23; revised 1996). The study protocol was approved by the Animal Experimentation Ethics Committee of Zunyi Medical University, China (approval no. ZMUER2014-2-069).

Experiment grouping. Eighty normal SPF SD male rats were randomly divided according to their body weight into two groups corresponding to the duration of administration (3 and 6 days). Each group of rats was further divided into blank control (2 $\mathrm{ml} 5 \%$ sodium carboxymethyl cellulose solution), positive control of CYP inducer $(50 \mathrm{mg} / \mathrm{kg} /$ day rifampicin), and experimental groups (Xiaochaihu decoction: Low dose, $1.7 \mathrm{~g} / \mathrm{kg} / \mathrm{day}$; medium dose, $3.4 \mathrm{~g} / \mathrm{kg} /$ day; and high dose, $6.8 \mathrm{~g} / \mathrm{kg} / \mathrm{day}$ ), with eight rats in each group. These drug doses given to animals were selected primarily in reference to previous studies $(17,18)$ and pre-experimental results.

Intragastric administration and sample extraction. The corresponding treatments were administered to the rats in each group every morning. After the last dose, the rats were fasted for $24 \mathrm{~h}$ and euthanised by cervical dislocation. Animals were anesthetized via intraperitoneal injection with pentobarbital sodium $(60 \mathrm{mg} / \mathrm{kg})$ prior to cervical dislocation. The livers of the rats were excised, frozen in liquid nitrogen, and stored in a refrigerator at $-80^{\circ} \mathrm{C}$.

Quality control of Xiaochaihu decoction by HPLC. Quality control of the Xiaochaihu decoction was carried out by using high pressure liquid chromatography (HPLC) to determine baicalin content in Xiaochaihu decoction. HPLC method was adapted from previously established method (19). Briefly, by using an Agilent-1260 HPLC system (Agilent Technologies, Inc.) with a photodiode array detector, the analysis was achieved through a TSK gel ODS C18 (250x4.6 mm; $5 \mu \mathrm{m}$; Tosoh) column with mobile phase of methanol-water phosphoric acid (65:35:0.7) at a flow rate of $1.0 \mathrm{ml} / \mathrm{min}$. The detection wavelength was set as $280 \mathrm{~nm}$, the column temperature as $30^{\circ} \mathrm{C}$, and the injection volume as $5 \mu 1$.

Total RNA isolation and reverse transcription-quantitative $(R T-q) P C R$ assays. Total RNA was isolated from rat liver tissue using RNAiso Plus (Berkeley), and the concentration was quantified by spectrophotometer (A260/A280). The RNA was reverse transcribed into complementary DNA (cDNA) using a FlexCycler and the RevertAid First Strand cDNA Synthesis Kit (Thermo Fisher Scientific, Inc.), according to the manufacturer's instructions. Target gene expression was analysed via qPCR system (Bio-Rad Laboratories, Inc.) with SYBR Green PCR kit (Berkeley). The reactions for each of the target mRNAs consisted of 25- $\mu$ l volumes including $12.5 \mu \mathrm{l}$ TB Green Premix Ex Taq II (Takara Bio, Inc.), $1 \mu \mathrm{l}$ specific 
Table I. Primer sequences used for reverse transcription-quantitative PCR reactions.

\begin{tabular}{lll}
\hline Gene & \multicolumn{1}{c}{ Forward $\left(5^{\prime}\right.$ to $\left.3^{\prime}\right)$} & Reverse $\left(5^{\prime}\right.$ to $\left.3^{\prime}\right)$ \\
\hline CYP1A2 & aggtcaaccatgatgagaagcagtg & aggaggatggctaagaagaggaagac \\
CYP1B1 & gagagttggtggcagtgttggtg & ctcggcatcgtcgtggttgtac \\
CYP2D6 & gtgctgccttcgctgaccatag & tccagattcctcctcaagagtgtcc \\
CYP3A1 & cgttcaccagtggaagactcaagg & acttcttcacagggacaggtttgc \\
$\beta$-actin & caccegcgagtacaaccttc & cccatacccaccatcacacc \\
\hline
\end{tabular}

forward primer, $1 \mu \mathrm{l}$ specific reverse primer, $2 \mu \mathrm{l}$ cDNA and $8.5 \mu 1$ sterile water. A standard PCR amplification procedure was performed as follow: $95^{\circ} \mathrm{C}$ for $30 \mathrm{sec}$, followed by 40 cycles of $95^{\circ} \mathrm{C}$ for $5 \mathrm{sec}$ and $60^{\circ} \mathrm{C}$ for $30 \mathrm{sec}$. Analysis of each specimen for each target was repeated three times. Gene transcription was analyzed by the $2^{-\Delta \Delta \mathrm{Cq}}$ method (20) using $\beta$-actin expression as a reference. The forward and reverse primer sequences used in this study are shown in Table I.

Western blot analysis. The protein levels of Cypla2, 1b1, 2d6 and $3 a 1$ in rat livers were evaluated using western blotting assay. Briefly, total protein was extracted from rat liver tissue using RIPA lysis buffer (Beijing Solarbio Science \& Technology Co., Ltd.) containing with $1 \%$ proteinase inhibitor phenylmethylsulfonyl fluoride (PMSF). After centrifugation, the supernatant of the lysates was obtained. The protein concentration was quantified using a bicinchoninic acid (BCA) assay kit (Beijing Solarbio Science \& Technology Co., Ltd.). The protein samples $(50 \mu \mathrm{g})$ were added to per lane, and separated on $10 \%$ sodium dodecyl sulphate-polyacrylamide gels by electrophoresis and then transferred onto polyvinylidene difluoride (PVDF) membranes. The membranes were blocked with 5\% non-fat milk at room temperature for $2 \mathrm{~h}$. The primary antibodies GAPDH (1:5,000, cat. no. BL006B; Biosharp life sciences Co., Ltd), CYP1A2 antibody (1:4,000, cat. no. bs-2589R; Biosynthesis Biotechnology Co., Ltd.), CYP1B1 antibody (1:4,000; cat. no. bs-12926R; Biosynthesis Biotechnology Co., Ltd.), CYP3A1 antibody (1:4,000, cat. no. bs-20586R; Biosynthesis Biotechnology Co., Ltd.) and CYP2D6 (1:4,000, cat. no. bs-1725R; Biosynthesis Biotechnology Co., Ltd.) were incubated at $4^{\circ} \mathrm{C}$ overnight. Goat anti-rabbit $\operatorname{IgG}$ secondary antibody (1:5,000; cat. no. BL003A; Biosharp Life Sciences) was added to the membranes and incubated for $1 \mathrm{~h}$ at room temperature. The protein bands were visualized using the ECL prime detection reagent (cat. no. KGP1121-KGP1123; Nanjing KeyGen Biotech Co., Ltd.) and using a ChemiDoc MP imaging system (Bio-Rad Laboratories, Inc.). Image Lab version 6.0 gel imaging system software (Bio-Rad Laboratories, Inc.) was used to analyze the gray value of the target strip.

Statistical analysis. SPSS20.0 (IBM Corp.) was used for statistical analysis of all data; and the Kolmogorov-Smirnov test was used as a distribution normality test. The comparison between groups was evaluated using one-way analysis of variance (ANOVA). When variance was equal, the last significant difference method was used for multiple comparisons between groups. When assessed variance was not equal, the Dunnett's T3 method was used for multiple comparisons between groups.
Table II. Content of baicalin in Xiaochaihu decoction $(n=3)$.

\begin{tabular}{lcc}
\hline Batch no. & Content, $\mathrm{mg} / \mathrm{ml}$ & Average content, $\mathrm{mg} / \mathrm{ml}$ \\
\hline 1 & 3.21 & \\
2 & 3.27 & 3.26 \\
3 & 3.30 & \\
\hline
\end{tabular}

Results are presented as mean $\pm \mathrm{SE}$. $\mathrm{P}<0.05$ was considered to indicate a statistically significant difference.

\section{Results}

Quality control of Xiaochaihu decoction. The HPLC chromatograms of reference substance and baicalin in Xiaochaihu decoction are presented in Fig. 1, and the retention time of baicalin in reference substance and in Xiaochaihu decoction were 4.87 and $4.85 \mathrm{~min}$, respectively. As shown in Table II, the average content of baicalin in Xiaochaihu decoction was $3.26 \mathrm{mg} / \mathrm{ml}$, which was consistent with previous results $(3.39 \mathrm{mg} / \mathrm{ml})(19)$, and the decoction could be used for follow-up research.

Effects of Xiaochaihu decoction on the mRNA and protein expression of Cypla 2 in the liver. Fig. 2 shows the relative expression of Cypla $2 \mathrm{mRNA}$ in the liver tissue of each group. Compared with that in the control, Cypla 2 mRNA was downregulated in the rifampicin group after 3 days of administration $(\mathrm{P}<0.05)$ but slightly upregulated after 6 days of administration. The results showed that rifampicin had no significant effects on the expression of Cypla2 when administered for a relatively longer time. Liver-specific Cypla 2 mRNA and protein expression levels were significantly downregulated in the medium-dose treatment groups after 3 days compared with those in the control group $(\mathrm{P}<0.01)$. In the high-dose treatment group, there was a slight but insignificant increase in Cypla2 mRNA and protein expression after 3 days $(\mathrm{P}>0.05)$. After 6 days, Cypla 2 was upregulated in the medium-dose group and upregulated slightly in other groups $(\mathrm{P}>0.05)$. These results showed that short-term administration of Xiaochaihu decoction can slightly inhibit Cypla 2 expression in rats; however, this inhibitory effect disappeared with prolonged administration.

Effects of Xiaochaihu decoction on mRNA and protein expression of Cyp3al in the liver. Fig. 3 shows the relative expression of Cyp3al mRNA in the livers of each group. Cyp3al mRNA and protein levels in the liver were increased in 
A

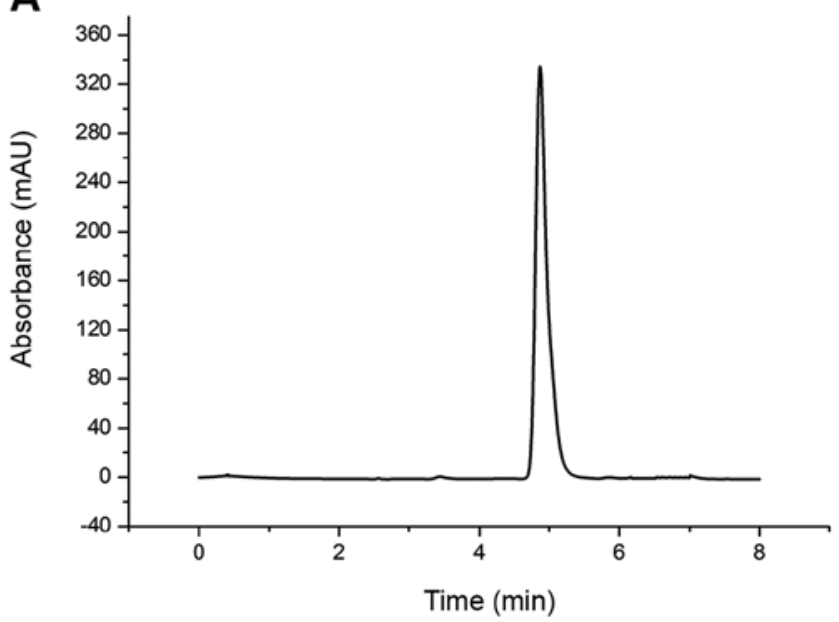

B

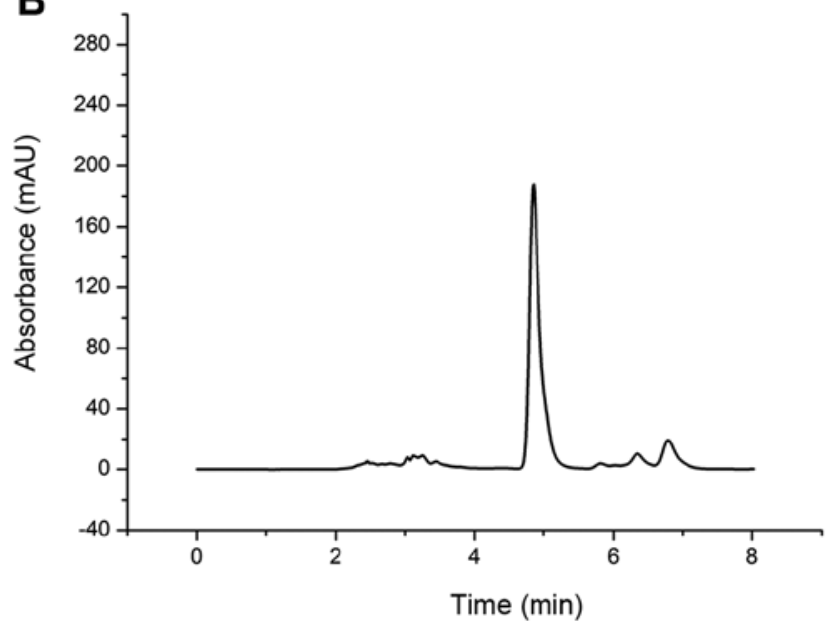

Figure 1. HPLC chromatogram of baicalin. Representative HPLC chromatogram of baicalin reference substance (A) and baicalin in Xiaochaihu decoction (B), with a retention time of 4.87 and $4.85 \mathrm{~min}$, respectively. HPLC, high-performance liquid chromatography.
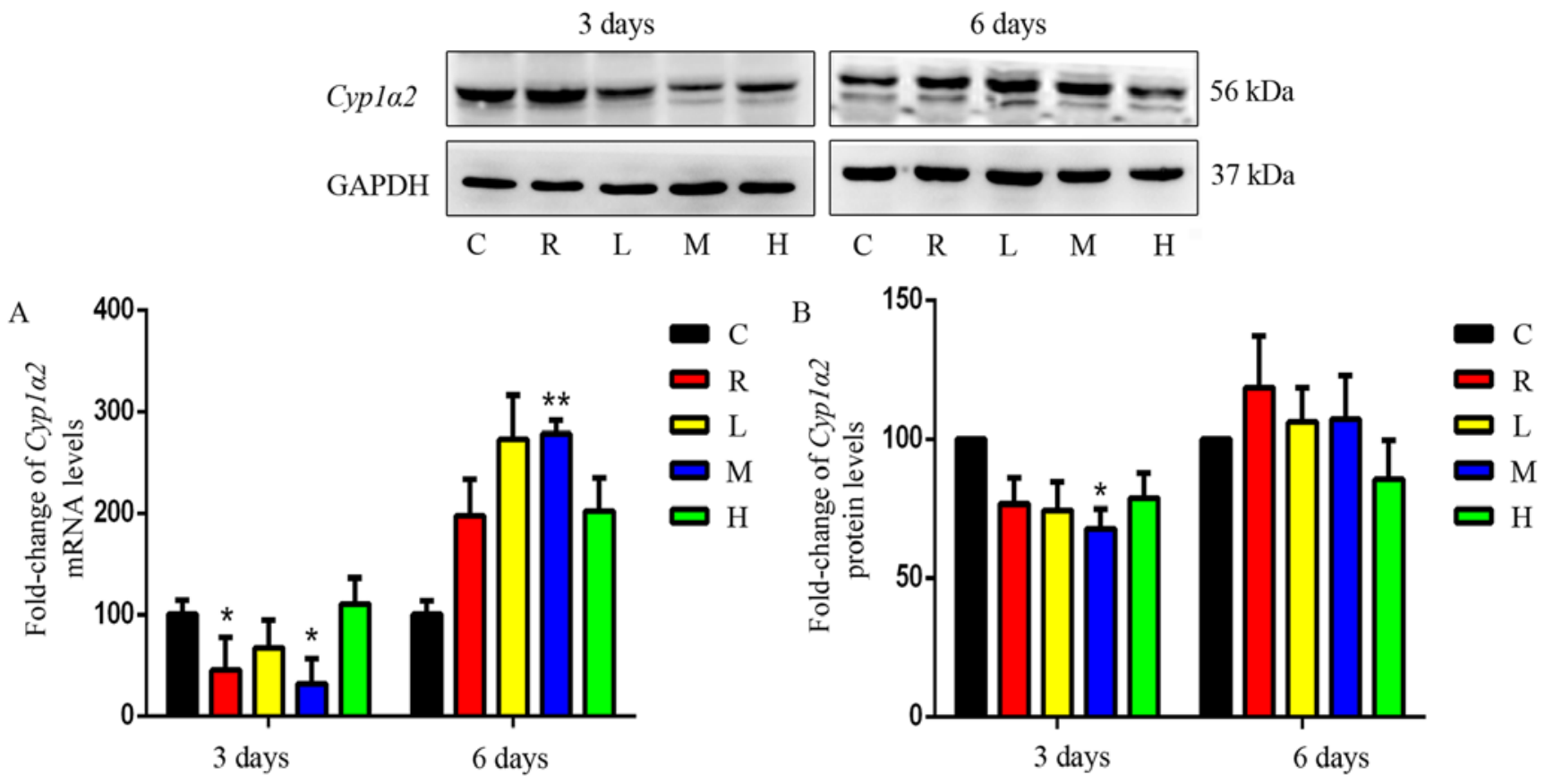

Figure 2. Relative expression level of Cypla2 mRNA and protein level. The relative expression level of Cypla2 mRNA (A) and protein (B) in the liver of rats which were treated with Xiaochaihu decoction once daily for 3 and 6 days. The data are expressed as mean $\pm \mathrm{SE}(\mathrm{n}=8)$. ${ }^{*} \mathrm{P}<0.05$; ${ }^{* *} \mathrm{P}<0.01 \mathrm{vs}$. control. C, control; $\mathrm{R}$, rifampicin; L, low-dose; M, medium-dose; and $\mathrm{H}$, high-dose group.

the rifampicin group after 6 days of administration compared with those in the control. There was a significant increase in Cyp3a1 mRNA expression in the medium-dose group compared with that in the low-dose group. Furthermore, there was a significant increase in Cyp $3 a 1$ mRNA expression in the high-dose group compared with that in the medium-dose group. Cyp $3 a 1$ protein levels were significantly higher in the high-dose group than in the low- and medium-dose groups. Thus, Xiaochaihu decoction was shown to induce Cyp $3 a 1$ mRNA and protein expression in a dose-dependent manner.

Effects of Xiaochaihu decoction on mRNA and protein levels of Cyp2d6 in the liver. As shown in Fig. 4, the differences in
Cyp2d6 mRNA or protein expression between the rifampicin or each Xiaochaihu group and the control after 3 days of administration were unchanged. After 6 days, Cyp $2 d 6$ mRNA and protein levels were mildly upregulated in the livers of the rifampicin group. Cyp2d6 mRNA was significantly upregulated in the livers of the low- and high-dose groups $(\mathrm{P}<0.05)$. Cyp $2 d 6$ protein levels were significantly upregulated in the livers of the high-dose group $(\mathrm{P}<0.05)$. Therefore, Xiaochaihu decoction induced Cyp $2 d 6$ mRNA and protein expression after administration for 6 days.

Effects of Xiaochaihu decoction on mRNA and protein expression of Cyplb1 in the liver. As demonstrated by Fig. 5, 

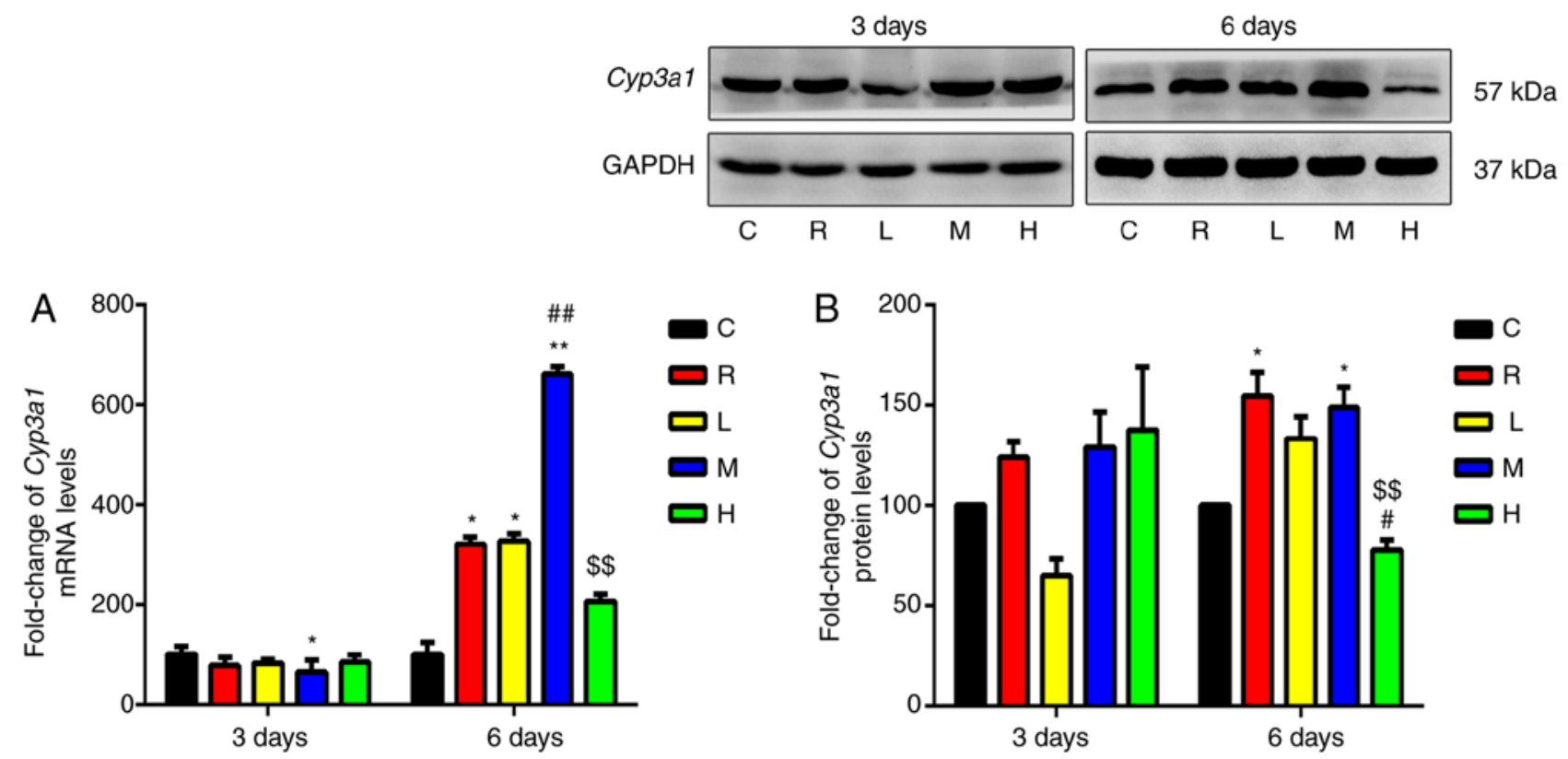

Figure 3. Relative expression level of Cyp3a1 mRNA and protein. Relative expression level of Cyp $3 a 1 \mathrm{mRNA}$ (A) and protein (B) in the liver of rats that were treated with Xiaochaihu decoction once daily for 3 and 6 days. The data are expressed as mean $\pm \mathrm{SE}(\mathrm{n}=8)$. " $\mathrm{P}<0.05$; ${ }^{* *} \mathrm{P}<0.01$ vs. control rats; ${ }^{*} \mathrm{P}<0.05$; ${ }^{\# \#} \mathrm{P}<0.01$ vs. Xiaochaihu decoction low-dose group; ${ }^{\$ \$} \mathrm{P}<0.01$ vs. Xiaochaihu decoction medium-dose group rats. C, control; R, rifampicin; L, low-dose; M, medium-dose; and $\mathrm{H}$, high-dose group.
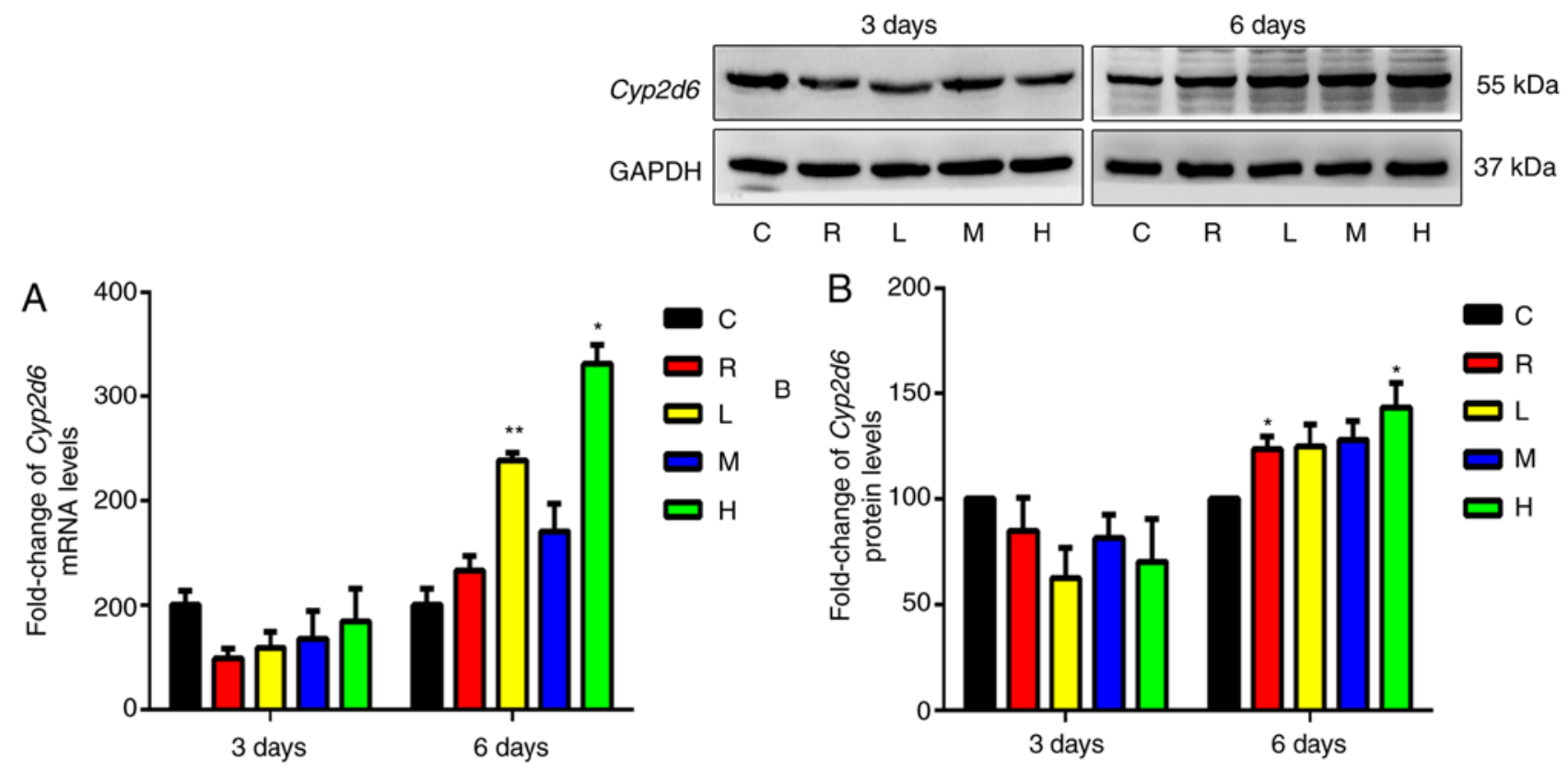

Figure 4. Relative expression level of Cyp2d6 mRNA and protein. The relative expression level of Cyp2d6 mRNA (A) and protein (B) in the liver of rats that were treated with Xiaochaihu decoction once daily for 3 and 6 days. The data are expressed as the mean $\pm \mathrm{SE}(\mathrm{n}=8)$. ${ }^{*} \mathrm{P}<0.05$; ${ }^{* *} \mathrm{P}<0.01 \mathrm{vs}$. control. C, control; $\mathrm{R}$, rifampicin; L, low-dose; M, medium-dose; and H, high-dose group.

the differences in Cyp1b1 mRNA or protein levels between the rifampicin for each Xiaochaihu group and the control were not significant $(\mathrm{P}>0.05)$. Xiaochaihu decoction may not affect the mRNA and protein expression of Cyplbl.

\section{Discussion}

Although several modern formulations of Xiaochaihu, including granules and tablets, have been developed from
Xiaochaihu decoction, and are widely used in clinical practice, the concentrated decoction is still administered in many cases. To better mimic the clinical use of drugs and consider the reference value of the research results for different forms of dosage, concentrated Xiaochaihu decoction was selected in the present study.

The main reasons for selecting the drug doses given to animals were selected primarily in reference to previous studies $(17,18)$; and pre-experimental results found that 

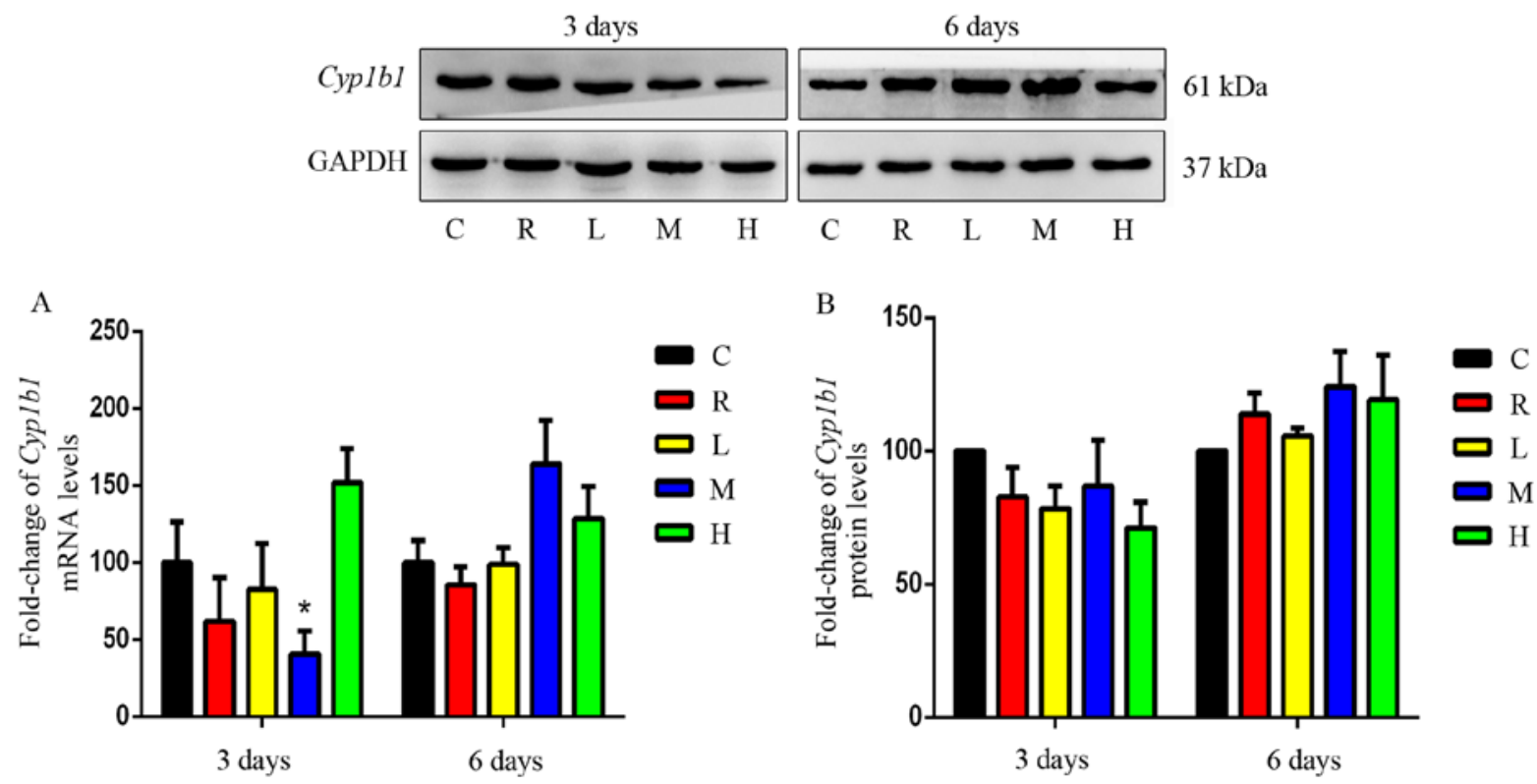

Figure 5. Relative expression level of Cyp1b1 mRNA and protein. The relative expression level of Cyp1b1 mRNA (A) and protein (B) in the liver of rats that were treated with Xiaochaihu decoction once daily for 3 and 6 days. The data are expressed as the mean $\pm \mathrm{SE}(\mathrm{n}=8)$. " $\mathrm{P}<0.05$ vs. control. C, control; R, rifampicin; L, low-dose; M, medium-dose; and H, high-dose group.

low-dose Xiaochaihu decoction has slightly stronger induction effect on CyplA2 and Cyp3A1 gene expression compared with medium and high dose at the doses of 13.6, 6.8 and $3.4 \mathrm{~g} / \mathrm{kg} /$ day. It was assumed that a lower dose of Xiaochaihu decoction may also have a certain induction effect on some CYPs, and the doses of Xiaochaihu decoction given to animals were decided as low dose of 1.7, medium dose of 3.4 and high dose $6.8 \mathrm{~g} / \mathrm{kg} / \mathrm{day}$ for subsequent experiments.

Scutellariae Radix is a main herb in the prescription of Xiaochaihu decoction. As a traditional Chinese herbal medicine, Scutellariae Radix exhibits important effects in the treatment of various diseases, including emesis, hepatitis and high blood pressure. Baicalin, the main bioactive ingredient in Scutellariae Radix, exerts antidepressant (21), anti-inflammatory and antioxidant effects (22). Therefore, baicalin content is determined for quality control of many traditional Chinese medicines containing Scutellariae Radix (23). In the Pharmacopoeia of the People's Republic of China 2015 edition, baicalin content was specified as a quality control parameter of Xiaochaihu granules (3). A quality control method for Xiaochaihu decoctions in clinical use and experimental research was established in the present study by referring to the quality control methods used for Xiaochaihu granules in the Pharmacopoeia of the People's Republic of China 2015 edition, and improving the relevant chromatographic conditions (19). Xiaochaihu decoction prepared using this method was uniform and quality was controllable.

Rifampicin is a broad-spectrum, classical, non-specific inducer of hepatic enzymes, including CYP3A, 1A2, 2B6, $2 \mathrm{C} 9,2 \mathrm{C} 19$ and 2D6, and rifampicin has the greatest effects on the expression of CYP3A in the liver (24). According to the US Food and Drug Administration, rifampicin can be used as a strong index inducer of CYP2B6 (moderate inducer), 2C8 (moderate inducer), 2C9 (moderate inducer), 2C19, and 3A when evaluating drug interactions (25). For the consideration of animal welfare (research on animals should involve the fewest number of animals) and simplicity in interpretation of results, only rifampicin was used as a positive drug for the different types of CYPs in the experiments. This experiment design can be found in some similar studies, in which only rifampicin or phenobarbital was used as a positive control for different CYPs $(26,27)$. However, it is reasonable that using a positive control for inhibition of hepatic enzymes may better reveal the different effects of Xiaochaihu decoction on different CYPs. The lack of a positive control for CYP inhibition is a limitation to the present study.

The present results revealed that rifampicin induced the gene and protein expression of Cyp $3 a 1$ after 6 days, and the difference was significant. This is consistent with the findings of a previous study in which rifampicin increased CYP3A4 expression (CYP3A4 in human was equivalent to CYP3A1 in rats) (28). After 3 days of rifampicin administration, the expression of Cypla 2 gene was inhibited; however, rifampicin had little effect on protein expression. The uncoordinated regulation of Cypla2 gene and protein expression in rats by rifampicin may be due to the regulation of protein expression at the transcriptional and translational levels. After 6 days of administration, rifampicin had little effect on the expression of Cyp2d6 gene and protein. These results were consistent with the findings by Rae et al (29) who showed that rifampicin had little effect on CYP2D6 gene expression in primary human hepatocytes. A previous study also found that rifampicin does not affect the gene and protein expression of Cyplb1 in rats (29). The present results indicated that rifampicin had varying degrees of effects on the genes and proteins of different subtypes of CYPs in rats, and that induction effects varied with the duration of administration.

Effect of Xiaochaihu decoction on the gene and protein expression of Cypla2 in rats. CYP1A2 accounts for $~ 13 \%$ 
of the total CYP enzyme content in the liver, making it the third most abundant CYP in the liver $(28,30)$. CYP1A2 gene and its human counterpart show a high homology of $80 \%$. Additionally, amino acid homology between CYP1A2 enzymes in humans and rats is 70\% (31). CYP1A2 mediates $10 \%$ of clinical drug metabolism (32), including in vivo elimination of propranolol, clomipramine, phenacetin, mexiletine, propanol, $\beta$-fluoroamine, verapamil and nifedipine $(13,33,34)$. As shown in Fig. 2, short-term administration of Xiaochaihu decoction inhibited the gene and protein expression of Cypla2 in rats. This was consistent with the result of the study by Saruwatari et al (35), in which Xiaochaihu decoction was found to have a slight inhibitory effect on human CYP1A2 activity using the probe drug method. It has also been reported that astragalus, baicalein and baicalin have an inhibitory effect on CYP1A2 activity (34-39). The inhibitory effect of Xiaochaihu decoction on the gene and protein expression of Cypla2 weakened with prolonged administration. It may be speculated that some components in Xiaochaihu decoction can slowly induce the expression of Cypla2 gene and protein. Liquorice (a component in Xiaochaihu decoction) can significantly increase the activity of CYP1A2 (39-41). The results showed that Xiaochaihu decoction had different effects on Cypla2 gene and protein expression at different durations of continuous administration. However, the inhibitory effects of short-term administration of Xiaochaihu decoction need to be considered when co-administered with the metabolic substrates of CYP1A2, such as clomipramine, phenacetin, and theophylline, since this inhibitory effect may increase the blood concentration of concomitant drugs, as well as the risks of poisoning and other ADRs.

Effect of Xiaochaihu decoction on the gene and protein expression of Cyp3al in rats. CYP3A4 is one of the most important CYPs and accounts for $60 \%$ of the total weight of CYP enzymes in human liver. It participates in the metabolism of $50 \%$ of all drugs (13), including macrolide antibiotics, imidazoles (antifungal medication), antivirals, rifamycins, selective serotonin reuptake inhibitors, calcium antagonists, $\beta$-hydroxy- $\beta$-methylglutaryl-CoA reductase inhibitors and benzodiazepines. Cyp3al in rats is equivalent to CYP3A4 in human. In the present study, short-term administration of low, medium and high doses of Xiaochaihu decoction showed slight inhibitory effects of varying degrees on the gene and protein expression of Cyp3al. Zhou et al (10) reported that a patient who had kidney transplantation in the previous year experienced CsA poisoning when Xiaochaihu granules were ingested concomitantly. Xiaochaihu granules were assumed to inhibit the gene and protein expression of CYP3A4, thus interfering with the metabolism of CsA and increasing its blood concentration. Xiaochaihu decoction may inhibit the expression of Cyp3al gene and protein because of the inhibitory effects of some of its components on Cyp3al. It has been reported previously that both baicalein and astragalus membranaceus extracts have inhibitory effects on CYP3A4 activity $(37,38)$.

After 6 days of continuous administration, low and medium doses of Xiaochaihu decoction induced Cyp3al gene expression, whereas the medium dose induced Cyp 3 al protein expression. The long-term use of Xiaochaihu decoction was found to induce Cyp3al gene and protein expression. This finding was consistent with the study by Nose et al (42), who showed the induction of Cyp3al gene by Xiaochaihu decoction in female SD rats. A possible reason for this induction is that Xiaochaihu decoction contains components that can induce the expression of Cyp3al gene and protein. As previously reported, saponins from Bupleurum can induce Cyp3al in mice. A high-dose Bupleurum injection $(0.72 \mathrm{ml} / \mathrm{kg})$ can induce the protein expression of CYP3A4 in mouse liver (43), and liquorice can significantly increase the enzymatic activity of CYP3A1/2 $(37,41,44)$. Therefore, when Xiaochaihu decoction is combined with the metabolic substrates of CYP3A1, such as macrolide antibiotics and imidazoles, the induction effects of Xiaochaihu decoction on CYP3A1 gene and protein expression should be considered.

Effect of Xiaochaihu decoction on the gene and protein expression of Cyp2d6 in rats. Although CYP2D6 only accounts for $2-4 \%$ of the total CYP content in the liver, it is involved in $20-25 \%$ of drug metabolism $(45,46)$. It can metabolise more than 30 drugs, such as dextromethorphan, statin, propranolol, codeine, selective serotonin reuptake inhibitors, antiarrhythmic drugs and antipsychotic drugs (47). In the present study, after 6 days of continuous administration, high-dose Xiaochaihu decoction induced Cyp2d6 gene and protein expression. It is suggested that Xiaochaihu decoction may slowly and dose dependently induce Cyp2d6 expression in rats through its ingredients. Consistent with this finding, a previous study reported that Xiaochaihu decoction can induce the expression of Cyp2d6 (48). Thus, when Xiaochaihu decoction is combined with the metabolic substrates of CYP2D6, such as isoquinoline, dextromethorphan, propranolol, statin, metoprolol and codeine, the induction of CYP2D6 expression should be considered.

Effect of Xiaochaihu decoction on the gene and protein expression of Cyplb1 in rats. CYP1B1 accounts for $3 \%$ of drug metabolism and is involved in the metabolic activation of $11 \%$ of precancerous carcinogens, such as polycyclic aromatic hydrocarbons (32). It can affect the tumour sensitivity of several chemotherapeutic drugs, such as paclitaxel, docetaxel and cyclophosphamide (49). The results of the present study showed that Xiaochaihu decoction does not affect the expression of Cyplbl gene and protein in normal rats. Thus, the effects of Xiaochaihu decoction on metabolic enzymes may be not be relevant for drugs metabolised by CYP1B1.

Conclusions. The study revealed that Xiaochaihu decoction can affect the expression of Cypla2, Cyp2d6 and Cyp3al in rats in a dose- and/or time-dependent manner. Moreover, it was found that there may be potential drug interactions when Xiaochaihu decoction is concomitantly administered with substrates of Cypla2, Cyp2d6 and Cyp3al (CYP1A2, CYP2D6 and CYP3A4 in humans).

\section{Acknowledgements}

The authors thank Ms Xue Lan in Zunyi Medical University for Carrying out the quality control of the Xiaochaihu decoction. 


\section{Funding}

This study was supported by the Science and Technology Foundation of Guizhou of China (grant no. Qian Kehe J word [2013] 2323), National Natural Science Foundation of China (grant no. 31460246), 2011 Collaborative Inovation Center of Guizhou Traditional Chinese Medicine and Ethnic medicine (grant no. Qianjiaokeyanfa [2012] 311), the Education Department of Guizhou Province of China (grant nos. GNYL[2017]006 and YLXKJS-YS-05).

\section{Availability of data and materials}

The datasets used and/or analyzed during the current study are available from the corresponding author on reasonable request.

\section{Authors' contributions}

FT, YT and HL conceived and designed the experiments; YT, HL, WW performed the experiments; HL, FT, CY analyzed the data; and FT, HL and YT prepared the manuscript. All authors read and approved the final manuscript.

\section{Ethics approval and consent to participate}

The present study was approved by the Animal Experimentation Ethics Committee of Zunyi Medical University of China (approval no. ZMUER2014-2-069).

\section{Patient consent for publication}

Not applicable.

\section{Competing interests}

The authors declare that they have no competing interests.

\section{References}

1. Nishimura N, Uemura T, Iwamoto K and Naora K: Change in tolbutamide permeability in rat jejunum and Caco-2 cells by Sho-saiko-to (Xiao Chai Hu Tang), a Chinese traditional medicine. J Pharm Pharmacol 62: 651-657, 2010.

2. Li JX, Cao Y, Zhang YW and Yang L: Analysis of disease spectrum of Xiaochaihu decoction. J China Pres Drug 16: 102-103, 2018 (In Chinese).

3. Chinese Pharmacopoeia Commission: Pharmacopoeia of the People's Republic of China: One. Beijing: China Medical Science Press, 2015: 574-576.

4. Liu CG and Du DH: Effects of Xiaochaihu decoction combined with Lansoprazole on reflux esophagitis. Contemp Med 21: $157-158,2015$

5. Li WJ: Evaluation of the efficacy of Xiaochaihu decoction in the treatment of angina pectoris. Res Integr Tradit Chin West Med 8: 41-42, 2016 (In Chinese).

6. Lin JB, Zhang YG and Lin PJ: To analyze the clinical effect of Xiaochaihu decoction combined with entecavir on chronic hepatitis B. Guide China Med 15: 204-205, 2016 (In Chinese).

7. Palleria C, Di Paolo A, Giofrè C, Caglioti C, Leuzzi G, Siniscalchi A, De Sarro G and Gallelli L: Pharmacokinetic drug-drug interaction and their implication in clinical management. J Res Med Sci 18: 601-610, 2018.

8. Nishimura N, Uemura T, Iwamoto K and Naora K: Effects of Sho-saiko-to on the pharmacokinetics and pharmacodynamics of tolbutamide in rats. J Pharm Pharmacol 50: 231-236, 1998.
9. Ohnishi N, Okada K, Yoshioka M, Kuroda K, Nagasawa K, Takara K and Yokoyama T: Studies on interactions between traditional herbal and western medicines. V. Effects of Sho-saiko-to (Xiao-Cai-Hu-Tang) on the pharmacokinetics of carbamazepine in rats. Biol Pharm Bull 25: 1461-1466, 2002.

10. Zhou Y, Chu XM, Ling SS, Cai WM and Wang QW: Effects of oral administration of Xiaochaih granule on whole blood cyclosporin A concentration in kidney transplantation patients. Chin J Hosp Pharm 19: 713-714, 1999 (In Chinese).

11. Khojasteh SC, Driscoll JP, Jackson KD, Miller GP, Mitra K, Rietjens IMCM and Zhang D: Novel advances in cytochrome p450 research. Drug Discov Today 16: 793-799, 2011.

12. Pillai VC, Strom SC, Caritis SN and Venkataramanan R: A sensitive and specific Cyp cocktail assay for the simultaneous assessment of human cytochrome p450 activities in primary cultures of human hepatocytes using LC-MS/MS. J Pharm Biomed Anal 74: 126-132, 2013.

13. Zanger UM and Schwab M: Cytochrome p450 enzymes in drug metabolism: Regulation of gene expression, enzyme activities, and impact of genetic variation. Pharmacol Ther 138: 103-141, 2013.

14. Zanger UM, Turpeinen M, Klein K and Schwab M: Functional pharmacogenetics/genomics of human cytochromes p450 involved in drug biotransformation. Anal Bioanal Chem 392: 1093-1108, 2008

15. Xiu LL, Yu X, Liu DN, Wang SR, Liu HY, Chen SH and Chen F: Effects of different species of sargassum and radix Glycyrrhizae in Haizao Yuhu decoction on cytochrome P450 in goiter rats. China J Trad Chin Med Pharm 32: 2020-2025, 2017 (In Chinese).

16. Cai H, Zhuang YS, Liu X and Cai BC: Orthogonal optimization of extraction technology of Xiaochaihu decoction. J Chin Med Mater 35: 2036-2039, 2012 (In Chinese).

17. Li J, Xie M and Gan Y: Effect of Xiaochaihu decoction and different herbal formulation of component on inhibiting H22 liver cancer in mice and enhancing immune function. Zhongguo Zhong Yao Za Zhi 33: 1039-1044, 2008 (In Chinese).

18. Wu FR, Ning LJ and Zhou R: Protective effect of Xiaochaihu decoction chemical hepatic fibrosis in mice. Chin J Clin Pharm Ther 25: 481-488, 2020 (In Chinese).

19. Lan X, Tang FS, Chen ZG, Zhang Y, Wang W and Zhou XM: Determination of baicalin in Xiaochaihu decoction by HPLC. J Zunyi Med Univ 38: 435-438, 2015 (In Chinese).

20. Livak KJ and Schmittgen TD: Analysis of relative gene expression data using real-time quantitative PCR and the 2(-Delta Delta C (T)) method. Methods 25: 402-408, 2001.

21. Zhao F, Tao W, Shang Z, Zhang W, Ruan J, Zhang C, Zhou L, Aiello H, Lai H and Qu R: Facilitating granule cell survival and maturation in dentate gyrus with Baicalin for antidepressant therapeutics. Front Pharmacol 11: 556845, 2020.

22. Yang F, Feng C, Yao Y, Qin A, Shao H and Qian K: Antiviral effect of baicalin on Marek's disease virus in CEF cells. BMC Vet Res 16: 371, 2020.

23. Li F, Wang WQ and You PJ: Advances in extraction technology of flavonoids from radix astragali. Mod Chin Med 12: 5-9, 2010 (In Chinese).

24. Niemi M, Backman JT, Fromm MF, Neuvonen PJ and Kivistö KT: Pharmacokinetic interactions with rifampicin: Clinical relevance. Clin Pharmacokinet 42: 819-850, 2003.

25. Huang SM, Temple R, Throckmorton DC and Lesko LJ: Drug interaction studies: Study design, data analysis, and implications for dosing and labeling. Clin Pharmacol Ther 81: 298-304, 2007.

26. Mo CK, Xie YR, Chen YL, Huang M and He F: The effect of Ginkgolide A on the expression of CYP450mRNA by application of human primary hepatocyte. Pharm Today 29: 79-82, 2019 (In Chinese).

27. Li Q, Zhen YQ, Cao WL, Gao L, Wang YC, Zhang XJ and Niu LY: Effects of saikosaponins and total glucosides of paeony on CYP450 enzyme activity and liver function of rat liver microsomes. Chin Tradit Pat Med 4: 3025-3028, 2019 (In Chinese).

28. He M, Huang RB and Liu Z: Effects of rifampicin on cytochrome P450 superfamily and related drugs. China Pharm 17: 1664-1666, 2006 (In Chinese).

29. Rae JM, Johnson MD, Lippman ME and Flockhart DA: Rifampin is a selective, pleiotropic inducer of drug metabolism genes in human hepatocytes: Studies with cDNA and oligonucleotide expression arrays. J Pharmacol Exp Ther 299: 849-857, 2001.

30. Takeda K, Watanabe J, Inoue K and Kanamura S: Rifampicin suppresses hepatic cyp2el expression and minimizes DNA injury caused by carbon tetrachloride in perivenular hepatocytes of mice. Alcohol Clin Exp Res 24 (Suppl 4): 87S-92S, 2000. 
31. Yue J, Peng RX, Yang J, Kong R and Liu J: CYP2E1 mediated isoniazid-induced hepatotoxicity in rats. Acta Pharmacol Sin 25: 699-704, 2004.

32. Wu S, Yang HL, Li H and Sun ZX: Prohibitive effect of processed polygonum multiflorum on CYP1A2 activities and mRNA expressions of rats. World Chin Med 11: 475-478-482, 2012 (In Chinese)

33. Pragyan P, Kesharwani SS, Nandekar PP, Rathod V and Sangamwar AT: Predicting drug metabolism by CYP1A1, CYP1A2, and CYP1B1: Insights from MetaSite, molecular docking and quantum chemical calculations. Mol Divers 18: 865-878, 2014.

34. Zhou SF, Wang B, Yang LP and Liu JP: Structure, function, regulation and polymorphism and the clinical significance of human cytochrome P4501A2. Drug Metab Rev 42: 268-354, 2010

35. Saruwatari J, Nakagawa K, Shindo J, Nachi S, Echizen H and Ishizaki T: The in-vivo effects of sho-saiko-to, a traditional Chinese herbal medicine, on two cytochrome P450 enzymes (1A2 and $3 \mathrm{~A}$ ) and xanthine oxidase in man. J Pharm Pharmacol 55: $1553-1559,2003$

36. Wei LY, Zhang YJ, Wei BH, Ye J, Yang XY and Sun GX: Effects of Coptis chinensis Franch, Scutellaria baicalensis Georgi and their compatibility on five CYP450 subenzymes in rat liver microsomes. China J Chin Mater Med 38: 1426-1429, 2013 (In Chinese).

37. Yao YC, Ni J, Han J, Feng LJ and Yan L: Effects of Scutellaria extract on cytochrome P450 enzymes. Chin J Exp Tradit Med Formul 18: 202-205, 2012 (In Chinese).

38. Tian S, He GR, Gao M, Sun JL, Wang FB and Gui GH: Drug interaction potential of oral baicalein on metabolic enzymes in rats. Chin J New Drug 21: 2230-2234+2301, 2012 (In Chinese).

39. Huang K, Liu ZH and He Q: Inhibition of Baicalin on activity of cytochrome P450 enzyme in rat and human liver microsomes. Chin J Exp Tradit Med Formul 22: 20-23, 2016 (In Chinese).

40. He YJ, Shi SY, Jin KT and Gao Y: The modulation effect of Glycyrrhiza in combination with Euphorbia pekinensis, Euphorbia kansui, and Daphne genkwa on the enzyme activity of CYP1A2 in rat livers. Chin Remed Clin 7: 278-280, 2016 (In Chinese)
41. Xiao CR, Wang YG, Dai FG, Ma ZC, Tan HL and Gao Y: The effect of aconitum coadministration with lilac daphne on cytochrome P450 in rat liver. Chin J Exper Tradit Med Formul 12: 48-50, 2006 (In Chinese).

42. Nose M, Tamura M, Ryu N, Mizukami H and Ogihara Y: Sho-saiko-to and Saiko-keisi-to, the traditional Chinese and Japanese herbal medicines, altered hepatic drug-metabolizing enzymes in mice and rats when administered orally for a long time. J Pharm Pharmacol 55: 1419-1426, 2003.

43. Wang YH, Qi JF and Lin M: Effect of total saikosaponins on the intestinal first-pass effect and liver Cyp3a,Cyp2el. Chin J Clin Pharm Ther 16: 740-748, 2011 (In Chinese).

44. Cheng Y, Huang Y, Tian Y, Xu L, Liu GQ and Zhang ZJ: Assessment of the effects of Radix Bupleuri and vinegar-baked Radix Bupleuri on cytochrome 450 activity by a six-drug cocktail approach. Chin J Nat Med 11: 302-308, 2013 (In Chinese).

45. Ingelman-Sundberg M: Genetic polymorphisms of cytochrome P450 2D6 (CYP2D6): Clinical consequences, evolutionary aspects and functional diversity. Pharmacogenomics J 5: 6-13, 2005.

46. Kawakami M, Takenoshita-Nakaya S, Takeba Y, Nishimura Y, Oda M, Watanabe M, Ohta Y, Kobayashi S, Ohtsubo T, Kobayashi S, et al: Evaluation of CYP2D6 protein expression and activity in the small intestine to determine its metabolic capability in the Japanese population. Biol Pharm Bull 40: 1344-1351, 2017.

47. Zhou SF, Di YM, Chan E, Du YM, Chow VD, Xue CC, Lai X, Wang JC, Li CG, Tian M and Duan W: Clinical pharmacogenetics and potential application in personalized medicine. Curr Drug Metab 9: 738-784, 2008.

48. Dai GF, Luo R, Wang YG, Xia CY, Tan HL, Xiao CR, Zhao YH and GaoY: Compatible effects of euphorbia and glycyrrhiza on CYP2E1 expression and activity in rat liver. J Third Mil Med Univ 27: 742-744, 2009 (In Chinese).

49. Liu MC, Meng QQ, Cui JH and Li JS: Recent research progress on the CYP1B1 inhibitors. Prog Mod Biomed 17: 4190-4196, 2017 (In Chinese).

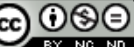

This work is licensed under a Creative Commons Attribution-NonCommercial-NoDerivatives 4.0 International (CC BY-NC-ND 4.0) License. 